\title{
Identification of $\gamma$-ray emission from 3C 345 and NRAO 512
}

F. K. Schinzel ${ }^{1, \star}$, K. V. Sokolovsky ${ }^{1,2, \star}$, F. D’Ammando ${ }^{3,4}$, T. H. Burnett ${ }^{5}$, W. Max-Moerbeck ${ }^{6}$, C. C. Cheung ${ }^{7}$, S. J. Fegan ${ }^{8}$, J. M. Casandjian ${ }^{9}$, L. C. Reyes ${ }^{10}$, M. Villata ${ }^{11}$, C. M. Raiteri ${ }^{11}$, I. Agudo ${ }^{12,13}$, O. J. A. Bravo Calle ${ }^{14}$, D. Carosati ${ }^{15,16}$, R. Casas ${ }^{17}$, J. L. Gómez ${ }^{13}$, M. A. Gurwell ${ }^{18}$, H. Y. Hsiao ${ }^{19}$, S. G. Jorstad ${ }^{12}$, G. Kimeridze ${ }^{20}$, T. S. Konstantinova ${ }^{14}$, E. N. Kopatskaya ${ }^{14}$, E. Koptelova ${ }^{19}$, O. M. Kurtanidze ${ }^{20}$, S. O. Kurtanidze Ku $^{20}$ V. M. Larionov ${ }^{21,22,14}$, E. G. Larionova ${ }^{4}$, L. V. Larionova ${ }^{14}$, A. P. Marscher ${ }^{12}$, D. A. Morozova ${ }^{14}$, M. G. Nikolashvili ${ }^{20}$, M. Roca-Sogorb ${ }^{13}$, J. A. $\operatorname{Ros}^{23}$, L. A. $\operatorname{Sigua}^{20}$, O. Spiridonova ${ }^{24}$, I. S. Troitsky ${ }^{14}$, V. V. Vlasyuk ${ }^{24}$, A. P. Lobanov ${ }^{1}$, and J. A. Zensus ${ }^{1}$

1 Max-Planck-Institut für Radioastronomie, Auf dem Hügel 69, 53121 Bonn, Germany e-mail: schinzel@mpifr-bonn.mpg.de

2 Astro Space Center of Lebedev Physical Institute, Profsoyuznaya Str. 84/32, 117997 Moscow, Russia

3 INAF-IASF Palermo, 90146 Palermo, Italy

4 INAF-IASF Roma, 00133 Roma, Italy

5 Department of Physics, University of Washington, Seattle, WA 98195-1560, USA

6 Cahill Center for Astronomy and Astrophysics, California Institute of Technology, Pasadena, CA 91125, USA

7 National Research Council Research Associate, National Academy of Sciences, Washington, DC 20001, resident at Naval Research Laboratory, Washington, DC 20375, USA

${ }^{8}$ Laboratoire Leprince-Ringuet, École polytechnique, Route de Saclay, 91128 Palaiseau, France

9 Laboratoire AIM, CEA-IRFU/CNRS/Université Paris Diderot, Service d'Astrophysique, CEA Saclay, 91191 Gif-sur-Yvette, France

10 Kavli Institute for Cosmological Physics, University of Chicago, Chicago, IL 60637, USA

11 INAF - Osservatorio Astronomico di Torino, 10025 Pino Torinese (TO), Italy

12 Institute for Astrophysical Research, Boston University, Boston, MA 02215, USA

13 Instituto de Astrofísica de Andalucía, CSIC, 18080 Granada, Spain

14 Astronomical Institute, St. Petersburg State University, St. Petersburg, Russia

15 EPT Observatories, Tijarafe, La Palma, Spain

16 INAF, TNG Fundación Galileo Galilei, La Palma, Spain

17 Institut de Ciencies de l'Espai (IEEC-CSIC), Campus UAB, 08193 Barcelona, Spain

18 Harvard-Smithsonian Center for Astrophysics, Cambridge, MA 02138, USA

19 Graduate Institute of Astronomy, National Central University, Jhongli 32054, Taiwan

20 Abastumani Observatory, Mt. Kanobili, 0301 Abastumani, Georgia

21 Isaac Newton Institute of Chile, St. Petersburg Branch, St. Petersburg, Russia

22 Pulkovo Astronomical Observatory, Pulkovskoe Chaussee 65/1, 196140 St. Petersburg, Russia

23 Agrupació Astronòmica de Sabadell, 08206 Sabadell, Spain

${ }^{24}$ Special Astrophysical Observatory, Nizhnij Arkhyz, Karachai-Cherkessian Republic, 369167, Russia

Received 15 November 2010 / Accepted 7 July 2011

\section{ABSTRACT}

For more than 15 years, since the days of the Energetic Gamma-Ray Experiment Telescope (EGRET) on board the Compton Gamma-Ray Observatory (CGRO; 1991-2000), it has remained an open question why the prominent blazar 3C 345 was not reliably detected at $\gamma$-ray energies $\geq 20 \mathrm{MeV}$. Recently a bright $\gamma$-ray source (0FGL J1641.4+3939/1FGL J1642.5+3947), potentially associated with 3C 345, was detected by the Large Area Telescope (LAT) on Fermi. Multiwavelength observations from radio bands to X-rays (mainly GASP-WEBT and Swift) of possible counterparts (3C 345, NRAO 512, B3 1640+396) were combined with 20 months of Fermi-LAT monitoring data (August 2008-April 2010) to associate and identify the dominating $\gamma$-ray emitting counterpart of 1FGL J1642.5+3947. The source 3C 345 is identified as the main contributor for this $\gamma$-ray emitting region. However, after November 2009 (15 months), a significant excess of photons from the nearby quasar NRAO 512 started to contribute and thereafter was detected with increasing $\gamma$-ray activity, possibly adding flux to 1FGL J1642.5+3947. For the same time period and during the summer of 2010, an increase of radio, optical and X-ray activity of NRAO 512 was observed. No $\gamma$-ray emission from B3 1640+396 was detected.

Key words. galaxies: active - quasars: individual: 3C 345 - gamma rays: galaxies - quasars: individual: NRAO 512 quasars: individual: B3 1640+396

\footnotetext{
* Member of the International Max Planck Research School (IMPRS) for Astronomy and Astrophysics at the Universities of Bonn and Cologne.
} 


\section{Introduction}

The quasar 3 C 345 is known as a prominent variable source from radio- to $\mathrm{X}$-ray bands $(\leq 10 \mathrm{keV})$. It is particularly bright at radio wavelengths and has an extended radio structure that is observable from sub-pc to kpc scales, which is archetypical for a relativistic blazar jet (e.g. Lobanov \& Zensus 1999; Kollgaard et al. 1989). Its jet has an apparent opening angle of $\sim 13^{\circ}$ (Pushkarev et al. 2009). Unwin et al. (1994) concluded that the observed high-energy emission of this source is caused by inverse Compton up-scattering of lower energy photons by relativistic electrons of the pc-scale radio jet. However, it remained a puzzle why 3C 345 was not detected at energies above $20 \mathrm{MeV}$, while 3C 279 with similar apparent properties is a prominent source at $\gamma$-ray energies.

The high-redshift quasar NRAO 512, $0.5^{\circ}$ west of $3 \mathrm{C} 345$, is known as a compact radio source with a two-sided jet morphology on kpc scales and with a flat radio spectrum (Kovalev et al. 1999). The observed VLBI radio structure revealed a wide apparent jet opening angle of $\sim 50^{\circ}$ (Pushkarev et al. 2009), which implies a small viewing angle to the line of sight, with its emission directly beamed at us.

The Energetic Gamma-Ray Experiment Telescope (EGRET; Thompson et al. 1993) on board the Compton Gamma Ray Observatory (CGRO) spacecraft did not observe a significant excess of $\gamma$-ray photons consistent with 3C 345 (3EG; Hartman et al. 1999), covering the first four phases of the CGRO mission (1991-1995). This lack of detectable flux of $\gamma$-rays above $20 \mathrm{MeV}$ from 3C 345 was attributed to the high detection threshold of EGRET. A source consistent with the position of 3C 345, labeled as EGR J1642+3940, was found by Casandjian $\&$ Grenier (2008). This source was seen almost entirely in a single two-week viewing period with an excess of $\sim 5.8 \sigma$, from April 23rd-May 7th, 1996 (vp5190), which was after the data used for the 3EG. Unfortunately, the angular resolution of EGRET was too poor to distinguish contributions from the other two nearby candidates NRAO 512 and B3 1640+396, see Table 1. Between October 1st and October 4th, 1993, a similar source was marginally detected by EGRET $(\sim 2.1 \sigma)$, but its centroid was localized closer to Mrk 501. In exactly the same viewing period, Kataoka et al. (1999) claimed EGRET detected a flare from Mrk 501, which lies $2.5^{\circ}$ east of 3C 345. This would then be the only time that either of those sources was seen by EGRET - a strange coincidence that led Casandjian \& Grenier to note that the EGR J1642+3940 source association with 3C 345 is "not clear".

On June 11th, 2008, the Fermi Gamma-ray Space Telescope (short Fermi) was launched, equipped with the Large Area Telescope (LAT) instrument (Atwood et al. 2009) providing continuous all-sky monitoring of $\gamma$-ray emission as a default mode of operation. The LAT, compared with EGRET, has an improved sensitivity by a factor of $\sim 25$ and an improved point-source localization. After 20 months of LAT operation within the region of interest, a localization improvement (comparing radii of $95 \%$ error circles) of a factor of $\sim 14$ was reached compared to EGRET. These improvements provided a new opportunity to probe 3C 345 for $\gamma$-ray emission above $100 \mathrm{MeV}$.

The three-month bright Fermi source list (OFGL) for the dataset collected between August and October 2008 reported a $\gamma$-ray source (OFGLJ1641.4+3939) near the position of 3C 345. It was then associated with the quasar B3 1640+396 (CLASS J1641+3935) at low confidence (Abdo et al. 2009b). The bright 0FGL source was listed in the first Fermi-LAT source catalog (1FGL) as 1FGL J1642.5+3947 (Abdo et al. 2010a),
Table 1. Radio positions and redshifts of candidate $\gamma$-ray counterparts.

\begin{tabular}{lccc}
\hline \hline Name(s) & 3C 345 & NRAO 512 & $\begin{array}{c}\text { B3 1640+396 } \\
\text { CLASS J1641+3935 }\end{array}$ \\
\hline RA & $16^{\mathrm{h}} 42^{\mathrm{m}} 58^{\mathrm{s}} .810$ & $16^{\mathrm{h}} 40^{\mathrm{m}} 29^{\mathrm{s}} .633$ & $16^{\mathrm{h}} 41^{\mathrm{m}} 47^{\circ} .540$ \\
Dec & $+39^{\circ} 48^{\prime} 36^{\prime \prime} 99$ & $+39^{\circ} 46^{\prime} 46^{\prime \prime} 03$ & $+39^{\circ} 35^{\prime} 03^{\prime \prime} .33$ \\
$z$ & 0.5928 & 1.666 & 0.540 \\
Refs. & $(1,3)$ & $(1,4)$ & $(2,5)$ \\
\hline
\end{tabular}

Notes. Name(s) - common source names. RA/Dec - right ascension/declination $(\mathrm{J} 2000)$ of the radio source localization. $z$ - source redshift.

References. The numerals in the references row translate to: (1) Fey et al. (2004); (2) Myers et al. (2003); (3) Marziani et al. (1996); (4) Stickel et al. (1989); (5) Brinkmann et al. (1995).

further constraining its localization. An association with 3C 345 was still not possible with high confidence based on Fermi-LAT data alone, nevertheless, 1FGL J1642.5+3947 was listed in the catalog as being affiliated with its most likely counterpart 3C 345 (Abdo et al. 2010b).

In October 2009 a GeV flare was detected from the vicinity of 0FGL J1641.4+3939 (Reyes \& Cheung 2009). Simultaneously, the GASP-WEBT collaboration reported on increased optical and radio activity of 3C 345 (Larionov et al. 2009). This event triggered additional observations at different frequencies, which made it possible to establish an identification through correlated multiwavelength activity.

Here we present 20 months of monitoring observations by Fermi-LAT of the region around 3C 345, NRAO 512 and B3 1640+396, as well as radio, optical, UV, and X-ray observations of these sources. Section 2 discusses the observations and data reduction methods applied to obtain calibrated datasets for the subsequent analysis. Section 3 discusses the results of Fermi-LAT observations and identification through multiwavelength counterparts of events in the light curve. Section 4 discusses and summarizes the findings of this paper.

\section{Observations and data reduction methods}

\section{1. $\gamma$-ray monitoring}

The Fermi-LAT (Atwood et al. 2009) is a pair-conversion telescope designed to cover the energy range from $20 \mathrm{MeV}$ to greater than $300 \mathrm{GeV}$. The 1FGL catalog, based on 11 months of monitoring data, lists the $\gamma$-ray source 1FGL J1642.5+3947 (RA $16^{\mathrm{h}} 42^{\mathrm{m}} 33.55^{\mathrm{s}}$, Dec $+39^{\circ} 47^{\prime} 38.8^{\prime \prime}$, 95\% position error radius $r 95=0.056^{\circ}$; Abdo et al. 2010a), which lies in close vicinity of the three quasars 3C 345, NRAO 512 and B3 1640+396, listed in Table 1.

The Fermi-LAT data on the field in which 1FGL J1642.5+ 3947 is located were processed and analyzed using the Fermi science tools software package (version: v9r15p $2^{1}$; Abdo et al. 2010a). The photons were extracted from a region of interest (ROI) centered on the radio position of $3 \mathrm{C} 345$ within a radius of $15^{\circ}$ and a time range of 20 months, August 4th, 2008, to April 4th, 2010 (in MET $239557417-292088627$ s). The general data processing procedures applied were similar to those described in Abdo et al. (2009b) and Abdo et al. (2010a).

\footnotetext{
1 http://fermi.gsfc.nasa.gov/ssc/data/analysis/ documentation/Cicerone/

2 Mission Elapsed Time (MET) is measured in seconds from 2001.0 UT, including leap seconds.
} 
To ensure that the collected events considered in this analysis have a high probability of being photons, the "diffuse class" selection was applied. Furthermore, events above the zenith angle $105^{\circ}$ were removed to avoid a significant contamination by $\gamma$ rays produced from cosmic-ray interactions in Earth's atmosphere $^{3}$ (Abdo et al. 2009c). The P6_V3_DIFFUSE instrument response functions (IRF) were used (see Rando et al. 2009).

The remaining $\gamma$-ray-like events were analyzed using a maximum-likelihood approach (Mattox et al. 1996) to localize $\gamma$-ray sources and extract their spectra. As in the analysis of 1FGL, two sets of standard LAT analysis tools were used, and their results compared for consistency. The first set, based on gtfindsrc and gtlike tools, employs an unbinned likelihood analysis in which the expected response of the LAT is evaluated separately for each photon, given its energy and direction with respect to the detector axes. The second toolset, pointlike, uses a simpler, binned analysis methodology, where photons are binned spatially and in energy, and an averaged set of response functions is applied. The two packages give very similar results, with the pointlike package running much more quickly.

In both likelihood methodologies the $\gamma$-ray emission in the ROI is assumed to arise from two diffuse components and a number of point sources. Each point source is modeled spatially as a delta function and spectrally as a power law. The statistical significance of a potential source is estimated by forming a "test statistic" $(T S)$,

$T S=2\left(\ln L_{1}-\ln L_{0}\right)$,

where $L_{1}$ is the value of the likelihood obtained in the "source" hypothesis, with the source present in the model of the ROI and $L_{0}$ is the value in the alternate, "null" hypothesis, that the source is not real (and hence not included in the ROI model). If the null hypothesis is true, and assuming certain applicability conditions are met, Wilks' theorem (Wilks 1938) states that $T S$ is distributed as $\chi^{2}(N)$, where $N$ is the number of additional parameters optimized in the source model leading to $L_{1}$. Because each point source has four free parameters (two for its position and two for its spectrum), we expect that $T S \sim \chi^{2}(4)$, from which its significance can be evaluated. A source-detection threshold of $T S=25$ was adopted in 1FGL, which corresponds to a false detection probability of $5 \times 10^{-5}$ if $T S \sim \chi^{2}(4)$.

The gtlike model used for localization in this paper includes the 1FGL J1642.5+3947 point-source component and all other point sources from the 1FGL catalog (Abdo et al. 2010b) within a $20^{\circ}$ radius around the radio position of $3 C 345$. Further analysis was performed using two point-source components instead of one to fit the observed data. The model parameters for sources outside the $15^{\circ}$ ROI were fixed to their respective 1FGL values. The model included 20 or 21 point sources with free parameters (flux and spectral index), depending on whether a single point source was fitted for 1FGL J1642.5+3947 (20 point sources) or whether two source components were added to take into account the separate contributions of 3C 345 and NRAO 512 (21 point sources). The background component of Galactic diffuse emission (gll_iem_vQ2.fit) and an isotropic component (isotropic_iem_vQ2.txt), both of which are the standard models available from the Fermi Science Support Center $(\mathrm{FSSC})^{4}$, were added as well. The isotropic

\footnotetext{
3 The Earth's limb lies at a zenith angle of $\sim 113^{\circ}$ at the $565 \mathrm{~km}$ nearly circular orbit of Fermi. The zenith angle is defined as the angle of a photon's apparent origin to the Earth-spacecraft vector.

4 http://fermi.gsfc.nasa.gov/ssc/data/access/lat/ BackgroundModels.html
}

component includes both the contribution from the extragalactic diffuse emission and from the residual, charged particle backgrounds.

To produce light curves, the standard tool gtlike was used, applying an unbinned spectral likelihood analysis as described above. A gtlike input source model was constructed based on the improved 20-month spectral fits obtained from the localization analysis. Only sources that were detected with a $T S>25$ in the 20-month integration were included in the final gtlike input source model to construct a light curve. The data were split into regular time intervals, each integrating over periods of 2,5 and 30 days. Then an unbinned spectral likelihood analysis was performed on each integrated dataset with photon indices fixed to the 20-month average values. For time periods with a significance of the detection of $T S<5$ on 5-and 30-day scales, $2 \sigma$ upper limits were calculated following the likelihood profile (Rolke et al. 2005), where the upper limit is determined by increasing the flux from the maximum likelihood value until the log likelihood decreases by 2.0 (for the particular implementation in case of LAT data see Abdo et al. 2010a). All light curves make use of the collected data in the energy range of $0.1-300 \mathrm{GeV}$. Different time binnings were chosen to study different aspects of the time evolution of the source flux. To study the longer term behavior, 30-day bins were used (Sect. 3.2.1), monthly bins being relatively standard for Fermi analysis. To assess faster flaring behavior, and in particular to estimate when the peak flaring occurs, a two-day time binning was selected (Sect. 3.2.2). Finally, the best compromise between short and long time bins is the light curve with five-day integration periods (Sect. 3.3).

\subsection{X-ray observations}

Triggered by the reported $\gamma$-ray flare of 0FGL J1641.4+3939 in October 2009 (Reyes \& Cheung 2009), X-ray observations of all three candidate counterpart sources were performed by the X-ray Telescope (XRT, 0.2-10 keV; Burrows et al. 2005) on board the Swift satellite (Gehrels et al. 2004). The list of Swift-XRT observations together with the spectral fit results is reported in Table 2.

The XRT data were processed with standard procedures (xrtpipeline v0.12.4) to calibrate the observations. The filtering and screening criteria were applied by means of the FTOOLS in the Heasoft package version $6.8^{5}$. Given the low fluxes of the sources during observations $\left(<0.5\right.$ counts $\mathrm{s}^{-1}$ in the $0.2-10 \mathrm{keV}$ range), only photon counting (PC) data were considered for our analysis with XRT grades 0-12 selected (according to Swift nomenclature, see Burrows et al. 2005). The source events were extracted in a circle of radius 15-20 pixels (covering $85-90 \%$ of the XRT point spread function, PSF, at $1.5 \mathrm{keV}$ ) around the source, depending on its intensity. The background was estimated from a nearby source-free circular region of 40-pixel radius. Ancillary response files were generated with xrtmkarf to account for different extraction regions, vignetting and PSF corrections. The spectral redistribution matrices v011 in the calibration database maintained by HEASARC were used.

The adopted energy range for spectral fitting is $0.3-10 \mathrm{keV}$. All data were rebinned with a minimum of 20 counts per energy bin to use the $\chi^{2}$ minimization fitting technique, except when the statistics were poor $(<200$ counts), in which case the

5 xrtpipeline and FTOOLS are part of the Heasoft software package: http://heasarc.gsfc.nasa.gov/lheasoft/; a detailed discussion on XRT data analysis can be found in the XRT User's Guide: http://heasarc.gsfc.nasa.gov/docs/ swift/analysis/xrt_swguide_v1_2.pdf 
Table 2. Swift-XRT observations after the reported flare in October 2009 of the three candidate sources (3C 345, NRAO 512 and B3 1640+396).

\begin{tabular}{lcccc}
\hline \hline Source & Obs. date $(*)$ & Exp. & Flux & Index \\
\hline 3C 345: & $2009-10-04(5109)$ & 4.2 & $6.8 \pm 0.5$ & $1.68 \pm 0.11$ \\
& $2009-10-06^{a}(5111)$ & 1.9 & $4.7 \pm 0.7$ & $1.66 \pm 0.17$ \\
& $2009-10-08(5113)$ & 2.1 & $7.1 \pm 0.8$ & $1.75 \pm 0.16$ \\
$2009-10-09(5114)$ & 2.2 & $6.4 \pm 0.7$ & $1.62 \pm 0.15$ \\
& $2009-10-18(5123)$ & 3.9 & $6.2 \pm 0.5$ & $1.67 \pm 0.12$ \\
& $2009-11-01(5137)$ & 4.1 & $5.5 \pm 0.5$ & $1.77 \pm 0.11$ \\
& $2009-11-15(5151)$ & 4.0 & $5.9 \pm 0.5$ & $1.88 \pm 0.11$ \\
& $2009-11-29(5165)$ & 4.1 & $5.7 \pm 0.5$ & $1.71 \pm 0.12$ \\
& $2010-03-06 / 07(5262 / 3)$ & 2.6 & $5.6 \pm 0.6$ & $1.84 \pm 0.15$ \\
& $2010-03-09^{a}(5265)$ & 1.4 & $5.6 \pm 0.8$ & $1.78 \pm 0.18$ \\
& $2010-04-09^{a}(5296)$ & 2.0 & $5.5 \pm 0.7$ & $1.88 \pm 0.16$ \\
NRAO 512: & $2010-08-18(5427)$ & 4.3 & $5.4 \pm 0.5$ & $1.54 \pm 0.11$ \\
& $2009-10-08^{a}(5113)$ & 2.1 & $0.6_{-0.2}^{+0.3}$ & $1.5 \pm 0.5$ \\
& $2009-10-15^{a}(5120)$ & 2.1 & $0.5 \pm 0.2$ & $2.2 \pm 0.6$ \\
B3 1640+396: & $2010-08-06 / 07^{a}(5415 / 6)$ & 5.5 & $0.7 \pm 0.1$ & $2.0 \pm 0.3$ \\
& $2009-12-01^{a}(5167)$ & 1.1 & $0.7+0.4$ & $1.8 \pm 0.7$ \\
\hline
\end{tabular}

Notes. Obs. date $(*)$ - date of Swift-XRT observation (*=JD2450000). Exp. - Swift-XRT effective exposure time in ks. Flux - integrated unabsorbed photon flux $0.3-10 \mathrm{keV}$ in units of $10^{-12} \mathrm{erg} \mathrm{cm}^{-2} \mathrm{~s}^{-1}$. (a) Cash statistic used for observations with less than 200 photons.

Cash statistic (Cash 1979) was used and the data were binned to 1 counts bin ${ }^{-1}$. The Cash statistic is based on a likelihood ratio test and is widely used for parameter estimation in photon counting experiments. Swift-XRT uncertainties are given at $90 \%$ confidence level for each parameter, unless stated otherwise.

Spectral fitting was performed using XSPEC version 12.5.1 with an absorbed power law model. For the Galactic absorption a fixed $\mathrm{H}_{\mathrm{I}}$ column density $n_{\mathrm{H}}$ of $1.14 \times 10^{20} \mathrm{~cm}^{-2}$ was used for $3 \mathrm{C} 345,1.17 \times 10^{20} \mathrm{~cm}^{-2}$ for $\mathrm{B} 31640+396$ and $1.07 \times 10^{20} \mathrm{~cm}^{-2}$ for NRAO 512, obtained from the Leiden/Argentine/Bonn Galactic Hi survey (Kalberla et al. 2005).

\subsection{UV, optical and $\mathrm{mm}$ observations}

In the case of 3C 345 a dense optical monitoring was performed by the GLAST-AGILE Support Program (GASP) of the Whole Earth Blazar Telescope (WEBT) collaboration. The GASP-WEBT is performing long-term monitoring of $28 \gamma$-ray loud blazars in the optical, near-infrared, $\mathrm{mm}$ and radio bands (Villata et al. 2008, 2009). The GASP has been closely following a strong optical outburst in 3C 345 reported during the second half of 2009 (Larionov et al. 2009). The optical GASP data for this paper were acquired at the following observatories: Abastumani (Georgia), Calar Alto (Spain, within the MAPCAT program), Crimean (Ukraine), Lowell (Perkins, USA), Lu-lin (SLT, Taiwan), Sabadell (Spain), St. Petersburg (Russia), and Tijarafe (Spain).

Magnitude calibration was performed with respect to a common choice of reference stars in the field of the source obtained from the photometric sequence by González-Pérez et al. (2001). In addition to the GASP monitoring data, optical observations performed by the Special Astrophysical Observatory (SAO) of the Russian Academy of Sciences, using the SAO Zeiss 1-m telescope in $B V R$ bands were included. The observational strategy and calibration procedures applied were described in detail by Bychkova et al. (2004). In Sect. 3.3 the $R$ band magnitudes were converted to the linear flux density scale to compare light curves between wavelengths. The magnitudes were corrected for a Galactic extinction of $0.036 \mathrm{mag}$, for the magnitude to flux conversion the zero magnitude fluxes of Bessell et al. (1998) were used.

Two $R$-band observations of 3 C 345 and B3 $1640+396$ were conducted on January 26th, $2009(14 \times 180 \mathrm{~s}$ exposure $)$, and on February 13th, $2009(13 \times 180 \mathrm{~s}$ exposure $)$, using a robotic 200-mm telescope of the Tzec Maun observatory (Mayhill, NM, USA). Magnitude calibration was performed in a manner similar to the GASP observations.

Observations of 3C 345 and NRAO 512 were performed with the UltraViolet/Optical Telescope (UVOT) on board the Swift spacecraft (Roming et al. 2005). Optical photometry of 3C 345 was calibrated using comparison stars from Smith et al. (1985). Calibration of the UV data was performed following the method of Poole et al. (2008). Comparison stars in the vicinity of 3C 345 could not be used to calibrate optical photometry of NRAO 512 with Swift UVOT because of its narrow field of view. Instead, the magnitude scale was calibrated using Sloan Digital Sky Survey (SDSS; Abazajian et al. 2009) stars in the field and transformations between the SDSS and Johnson photometric systems from Smith et al. (2002).

Table 3 lists the relevant results of mainly quasisimultaneous optical/UV observations of 3C 345, NRAO 512 and B3 1640+396 performed at Tzec Maun observatory and Swift UVOT. The Galactic extinction in the direction of these sources is between $E(B-V)=0.010 \mathrm{mag}$ and $0.014 \mathrm{mag}$ according to the tables of Schlegel et al. (1998). Using the extinction law by Cardelli et al. (1989) and coefficients describing the UVOT filters (Roming et al. 2009), the following extinctions (in magnitudes) were obtained for the individual bands: $0.03-0.04(V), 0.04-0.06(B), 0.05-0.07(U)$, $0.07-0.09(W 1), 0.09-0.13(M 2)$ and $0.08-0.12(W 2)$.

The source $3 \mathrm{C} 345$ was monitored also at $\mathrm{mm}$ wavelengths (230 GHz) by the Sub-millimeter Array (SMA) on Mauna Kea for the GASP. Additionally, a program conducted by Ann Wehrle (priv. comm.) provided the data collected by the SMA for NRAO 512 and B3 1640+396.

\section{Results}

\subsection{Localization of $\gamma$-ray emission}

An unbinned spectral likelihood analysis was performed using integrated 20-month Fermi-LAT monitoring data in the energy range of $0.1-100 \mathrm{GeV}$. This energy range was chosen after a series of different energy cuts were compared, to minimize the error on the localization. In the 1FGL cata$\log \gamma$-ray sources are typically localized with a $95 \%$ position error radius of $0.02^{\circ}-0.07^{\circ}$ for sources with $T S \approx 1000$, but $0.06^{\circ}-0.5^{\circ}$ for $T S \approx 30$ (Abdo et al. 2010a). In cases of faint $\gamma$-ray emitters it is difficult to obtain well-constrained localizations to make statistically significant associations, even more so for two faint $\gamma$-ray emitters in close vicinity of each other.

A single point-source, 1FGL J1642.5+3947, is listed in the 1FGL catalog around the analyzed region. Assuming a single point-source, the best-fit position using the 20month LAT dataset is RA $16^{\mathrm{h}} 42^{\mathrm{m}} 24^{\mathrm{s}}$, Dec $+39^{\circ} 48^{\prime} 27^{\prime \prime}$, with 
Table 3. Quasi-simultaneous optical observations of 3C 345 , NRAO 512 and B3 1640+396 conducted by Swift UVOT and the 200-mm telescope of Tzec Maun observatory.

\begin{tabular}{lccc}
\hline \hline Source & Obs. date $(*)$ & Band & Magnitude \\
\hline 3C 345: & $2009-01-26(4858)$ & $R$ & $16.48 \pm 0.02$ \\
& $2009-02-13(4876)$ & $R$ & $16.92 \pm 0.03$ \\
& $2009-10-08(5113)$ & $U$ & $16.44 \pm 0.06$ \\
& $2009-10-08(5113)$ & $U V W 1$ & $16.28 \pm 0.03$ \\
& $2010-08-18(5427)$ & $U V W 1$ & $16.47 \pm 0.02$ \\
NRAO 512: & $2009-10-08(5113)$ & $U$ & $18.61 \pm 0.04$ \\
& $2010-08-06(5415)$ & $U$ & $17.71 \pm 0.05$ \\
B3 1640+396: & $2010-08-06(5415)$ & $U V W 1$ & $18.07 \pm 0.02$ \\
& $2009-01-26(4858)$ & $R$ & $18.57 \pm 0.13$ \\
& $2009-02-13(4876)$ & $R$ & $>18.6^{\dagger}$ \\
\hline
\end{tabular}

Notes. Obs. date $(*)$ - date of Swift-XRT observation $(*=$ JD$2450000)$. Band - optical filter used for photometric observations. Magnitude - observed optical magnitude in given filter band. The magnitudes are not corrected for Galactic extinction. ${ }^{(\dagger)}$ No detection.

a $95 \%$ error circle radius of $0.037^{\circ}$. This procedure also provided the following characteristic source parameters: photon index ${ }^{6}: \Gamma=2.44 \pm 0.03$, integrated flux: $(1.78 \pm 0.08) \times$ $10^{-7} \mathrm{ph} \mathrm{cm}^{-2} \mathrm{~s}^{-1}, T S$ value of 2825 . The estimated systematic uncertainty of the integrated flux is $10 \%$ at $100 \mathrm{MeV}, 5 \%$ at $500 \mathrm{MeV}$ and $20 \%$ at $10 \mathrm{GeV}$. After 20 months of data the localized position is shifted by $0.042^{\circ}$ from its 1 FGL position toward NRAO 512, separated by $0.13^{\circ}$ (east) from 3C 345 and by $0.48^{\circ}$ (west) from NRAO 512. This suggested a significant contribution of excess photons from the direction west of 3C 345.

The unbinned spectral likelihood analysis was complemented by a pointlike analysis, similar to the method applied to determine positions and error ellipses for the 1FGL catalog. The approach of pointlike is described in Sect. 4.2 of Abdo et al. (2010a). This analysis revealed that a significant number of excess $\gamma$-ray photons were detected from the vicinity of NRAO 512 after November 2009 (15 month). A fit with two point-source models in which source positions were not fixed, representing contributions by 3C 345 and NRAO 512, yielded significant detections for the 20-month period (see Table 4). Figure 1 shows the radio positions of the three candidate sources together with the EGRET and 1FGL localizations, as well as the improved localizations for the two $\gamma$-ray sources found in the 20-month dataset. B3 $1640+396$ is $19.3^{\prime}$ and $16.5^{\prime}$ separated from the respective localizations of $3 \mathrm{C} 345$ and NRAO 512. Both $\gamma$-ray counterparts of 3C 345 and NRAO 512 now coincide with their respective radio positions (within the $95 \%$ error ellipse). This suggests that both sources may be $\gamma$-ray emitters. This is firmly established in Sect. 3.3 using the broadband temporal characteristics of the sources. There is no evidence for significant $\gamma$-ray emission from the third candidate source, B3 1640+396. The results of pointlike were cross-checked with an iterative use of gtlike and gtfindsrc, which confirm the pointlike results within errors, see Table 4. A $2 \sigma$ upper limit was calculated for B3 $1640+396$ by fixing each of the three candidate sources to their radio positions and assuming a $\Gamma=2.3$ spectral shape for B3 $1640+396$ and using the previously fitted $\Gamma$ for 3 C 345 and NRAO 512. This resulted in a 20-month upper limit for the integrated flux of $7.4 \times 10^{-9} \mathrm{ph} \mathrm{cm}^{-2} \mathrm{~s}^{-1}$. Figure 2 shows the observed binned spectra of the two sources together with their respective power-law fits. The pointlike

\footnotetext{
${ }^{6}$ The photon spectral index $\Gamma$ is defined as $N(E) \propto E^{-\Gamma}$, where $N(E)$ is the $\gamma$-ray photon flux as a function of energy $E$.
}

Table 4. The unbinned spectral likelihood results for the point-source localizations and spectra of 3C 345 and NRAO 512.

\begin{tabular}{lcc}
\hline \hline & $3 \mathrm{C} 345$ & NRAO 512 \\
\hline RA (J2000) & $16^{\mathrm{h}} 43^{\mathrm{m}} 0.24^{\mathrm{s}}$ & $16^{\mathrm{h}} 40^{\mathrm{m}} 44.4^{\mathrm{s}}$ \\
$\operatorname{Dec}(\mathrm{J} 2000)$ & $+39^{\circ} 48^{\prime} 22.7^{\prime \prime}$ & $+39^{\circ} 46^{\prime} 12.0^{\prime \prime}$ \\
$\Delta \mathrm{r}$ & $0.35^{\prime}$ & $2.9^{\prime}$ \\
$\mathrm{a}(95 \%)$ & $0.0490^{\circ}$ & $0.0735^{\circ}$ \\
$\mathrm{b}(95 \%)$ & $0.0442^{\circ}$ & $0.0626^{\circ}$ \\
$\Phi$ & 41.6 & -33.2 \\
$\Gamma(\mathrm{pl})$ & $2.49 \pm 0.02$ & $2.37 \pm 0.04$ \\
$\Gamma(\mathrm{gt})$ & $2.45 \pm 0.05$ & $2.41 \pm 0.08$ \\
Flux (pl) & $1.22 \pm 0.10$ & $0.51 \pm 0.23$ \\
Flux (gt) & $1.13 \pm 0.13$ & $0.67 \pm 0.13$ \\
Pivot Energy & 949 & 1598 \\
$T S$ & 1076 & 246 \\
\hline
\end{tabular}

Notes. RA/Dec - right ascension/declination of the $\gamma$-ray localization; $\Delta r$ - distance to respective radio source position; a $(95 \%)-$ major axis of $95 \%$ localization error ellipse; b (95\%) - minor axis of $95 \%$ localization error ellipse; $\Phi$ - rotation angle of the error ellipse East of North (degrees); $\Gamma$ - spectral index of power-law fit, $(\mathrm{pl})$ are the values obtained from the pointlike fit and (gt) are values from the gtlike fit; Flux - 20-month average flux $(0.1-100 \mathrm{GeV})$ determined from the power-law spectral fit in units of $10^{-7} \mathrm{ph} \mathrm{cm}^{-2} \mathrm{~s}^{-1}$; Pivot Energy in $\mathrm{MeV}$, this is the energy for which there is no correlation between the flux or normalization and spectral index uncertainties; $T S$ - likelihood test statistic value.

best-fit parameters are listed in Table 4. In Fig. 3 the respective 30-day binned $\gamma$-ray light curves of the two localized sources are shown and are discussed below in more detail.

\subsection{0-month of $\gamma$-ray monitoring}

\subsubsection{Long-term variability of $3 \mathrm{C} 345$ and NRAO 512}

To study the long-term variability, light curves were produced for the $\gamma$-ray counterparts associated with 3C 345 and NRAO 512 with 30-day binning. The 30-day binned light curve is shown in Fig. 3. For most of the data points NRAO 512 did not reach above a $T S$ of 25 , except for the last five months of the 20 -month period. This led to the conclusion that NRAO $512 \mathrm{did}$ not produce a significant excess of $\gamma$-ray emission over the local background during the first 15 months of LAT operation; thus it did not appear in the 1FGL catalog. After 15 months the amount of excess photons from the vicinity of NRAO 512 slowly increased, making a detection over the integrated 20 -month period possible. The average Fermi-LAT flux for the first 15-months of the monitoring period was $(0.42 \pm 0.07) \times 10^{-7} \mathrm{ph} \mathrm{cm}^{-2} \mathrm{~s}^{-1}$ compared to $(1.1 \pm 0.1) \times 10^{-7} \mathrm{ph} \mathrm{cm}^{-2} \mathrm{~s}^{-1}$ for the last quarter of the monitoring period (months $15-20$ ).

The observed $\gamma$-ray flux values reveal variability on timescales from days to months. To quantify this variability, the variability index $(V)$ as defined through the $\chi^{2}$ distribution (see Abdo et al. 2010a) was determined. It is computed from the 30-day integrated light curve data shown in Fig. 3. Whenever $T S<5$ the $2 \sigma$ upper limit was computed and its error estimate for that interval was replaced with half the difference between that upper limit and its value determined through the unbinned spectral likelihood analysis. In the absence of variability $V$ is expected to follow a $\chi^{2}$ distribution with $19\left(=N_{\text {int }}-1\right)$ degrees of freedom. At the $99 \%$ confidence level the light curve is significantly different from a flat one if $V>36.2$. For 3C 345 a $V$ of 115.3 was obtained, for NRAO 512 it was 37.7. Both 


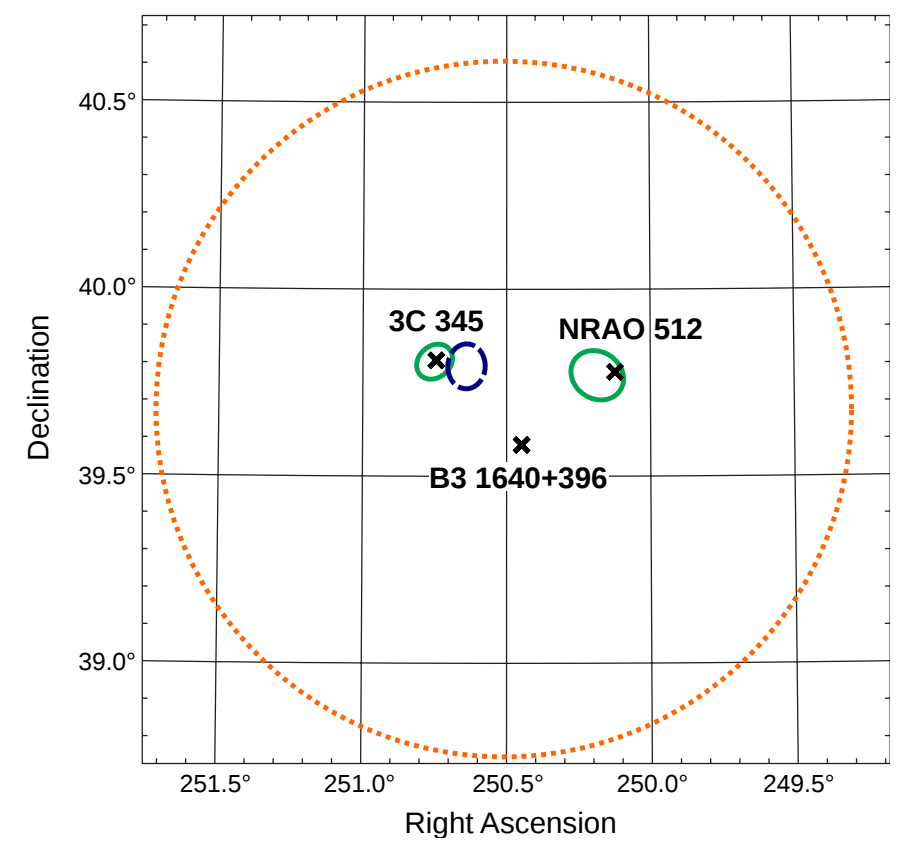

Fig. 1. $\gamma$-ray counterpart localizations together with the positions of the radio counterparts of the three candidate sources. Crosses mark the radio positions of $3 \mathrm{C} 345$, NRAO 512 and $\mathrm{B} 31640+396$. The large dotted orange circle denotes the $95 \%$ confidence error localization of EGR J1642+3940 (Casandjian \& Grenier 2008). The dashed blue ellipse denotes the $95 \%$ confidence error localization of 1FGL J1642.5+3947 (Abdo et al. 2010a). The two solid green ellipses denote the $95 \%$ confidence error localization of 3C 345 and NRAO 512 presented in this paper. The confusing source Mrk 501 is $2.5^{\circ}$ east (left) and $4 \mathrm{C}+38.41$ is $2.1^{\circ}$ southwest of the field center.

sources are variable according to the $99 \%$ confidence interval with higher variability observed from 3C 345.

In the shorter binned light curve of NRAO 512, 63\% of the derived values had a $T S<5$ (i.e. non-detections) compared to $\sim 48 \%$ for 3C 345 . Because NRAO 512 did not show a significant flux for most of the time on integrations of five days or less and with the observation that the flux of 3C 345 dominates, the model component of NRAO 512 was removed and light curves were produced with a single component fixed at the position of the $\gamma$-ray localization of 3C 345 listed in Table 4. This reduced the amount of noise in the light curve of 3C 345 and the number of values with a $T S<5$ was only $14 \%$.

\subsubsection{Short-term variability of $3 C 345$}

For the remainder of this paper the discussion is based on light curves produced with a single point-source model component. The five-day binned light curve of 3C 345 is shown in Fig. 4 for comparison with observations at other wavelengths.

To investigate shorter term variability in the observed $\gamma$-ray emission of 3C 345, several prominent light-curve "flare events" were identified and parameters such as rise time, fall time and time of the peak were extracted using the five-day and two-day integrated light curves. These parameters can be used to constrain the size of emission regions and cooling-times. Table 5 summarizes these results, listing characteristic parameters for all $\gamma$-ray flare events for which the peak had a significance of $T S>25$ in the two-day light curve. This corresponded to a flux greater than $3.0 \times 10^{-7} \mathrm{ph} \mathrm{cm}^{-2} \mathrm{~s}^{-1}$. The time of onset and end
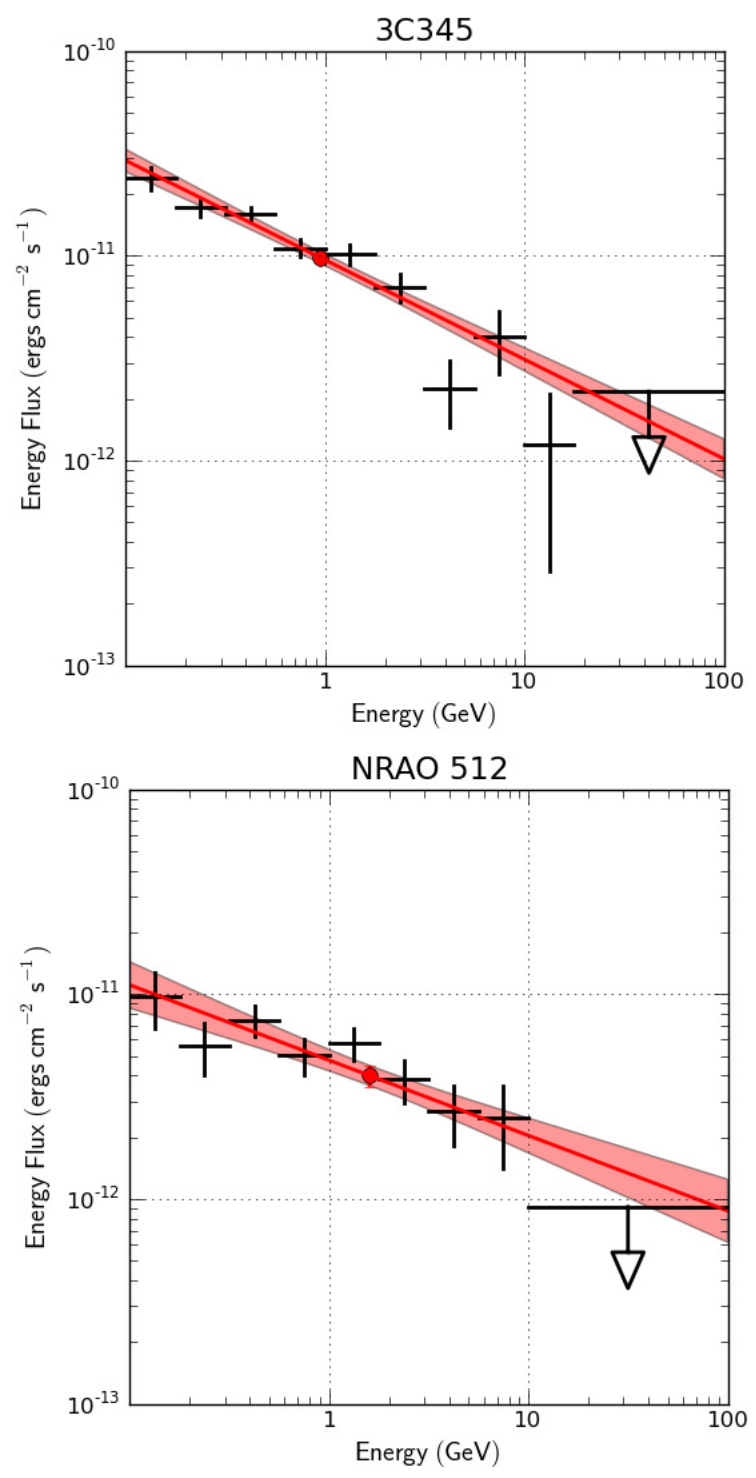

Fig. 2. Twenty-month $\gamma$-ray spectra of 3C 345 and NRAO 512. The red curve represents the obtained respective average power-law fits. The red dots correspond to the pivot energies listed in Table 4.

of an individual flare event was determined through a change in the sign of the gradient at the rising and declining slope of the light curve around the peak of a flare event.

Three dominant flare events of at least $5.0 \times$ $10^{-7} \mathrm{ph} \mathrm{cm}^{-2} \mathrm{~s}^{-1}$ and a significance of above $7 \sigma$ were observed on two-day time scales, one during 2008 and two during 2009. They were labeled with I-III in Fig. 4. Several weaker flares were identified and labeled with numbers from 1 to 6 . On average, the flares had a duration of 38 days a slightly faster rise than fall time and an average peak flux of $(5.0 \pm 0.5) \times$ $10^{-7} \mathrm{ph} \mathrm{cm}^{-2} \mathrm{~s}^{-1}$ (two-day integrations centered on the peak).

\subsection{Identification of $\gamma$-ray counterparts}

Following the $\gamma$-ray outburst on October 2nd, 2009, Swift observations were triggered on the candidate sources 3C 345, NRAO 512 and B3 1640+396. X-ray spectra and optical magnitudes were obtained from these observations. A summary of relevant measurements is listed in Tables 2 and 3. 
F. K. Schinzel et al.: Identification of $\gamma$-ray emission from 3C 345 and NRAO 512

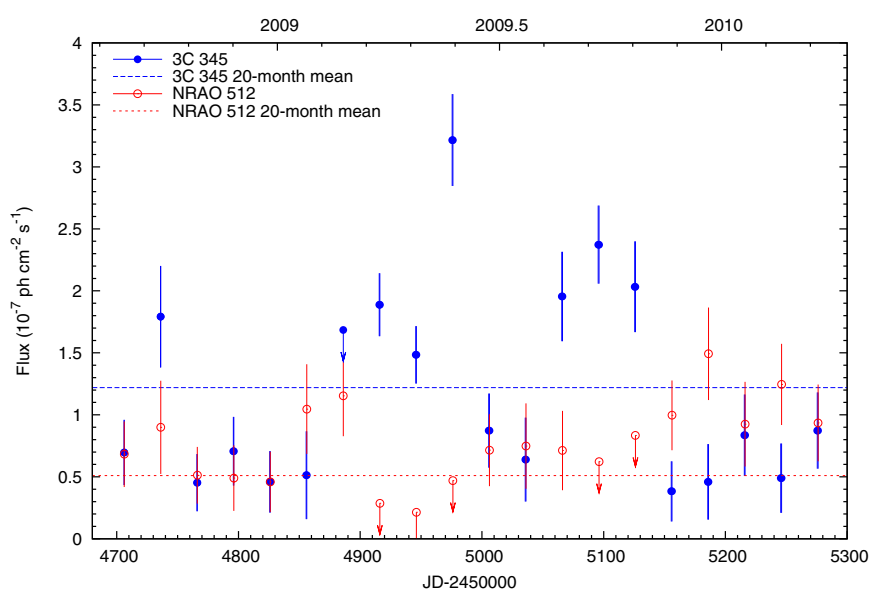

Fig. 3. $\gamma$-ray light curves of $3 \mathrm{C} 345$ and NRAO512 for the first 20 months of Fermi-LAT monitoring, obtained through fitting two point-source model components with power-law spectra to the region of interest placed at the respective counterpart positions. Values were determined from integrating observations over 30 days within an energy range of $0.1-300 \mathrm{GeV}$. Blue filled circles are values obtained for 3C 345, red open circles are values for NRAO 512. Values with downward arrows represent $2 \sigma$ upper limits shown in place of detections with low significance $(T S<5)$. The dashed lines plot the respective 20 -month average $\gamma$-ray source flux. Errors are statistical only.

The brightest source of the three candidates in X-ray as well as in optical is 3C 345. On January 26th, 2009, 3C 345 and B3 $1640+396$ were detected in the optical $R$-band at respective magnitudes of $16.48 \pm 0.02$ and $18.6 \pm 0.1$. On October 8th, 2009, observations of 3C 345 and NRAO 512 showed respective optical $U$-band magnitudes of $16.45 \pm 0.08$ and $18.64 \pm 0.04$. Both NRAO 512 and B3 1640+396 were a factor of 7-8 fainter than $3 \mathrm{C} 345$ at optical wavelengths. At X-ray energies, as seen from Table 2, NRAO 512 and B3 1640+396 had similar fluxes, which were a factor of 8-9 fainter than 3C 345. Archival Swift-UVOT and XRT data from observations of NRAO 512 made in January 2007 have an average optical $U$ magnitude of $18.0 \pm 0.1$ and an X-ray flux of $(8.3 \pm 2.3) \times$ $10^{-13} \mathrm{erg} \mathrm{cm}^{-2} \mathrm{~s}^{-1}$, which are consistent with the recently observed values. Quasi-simultaneous $\mathrm{mm}$ observations between October and December 2009 determined the fluxes of 3C 345, NRAO 512 and B3 1640+396 to be $\sim 3.0, \sim 0.4$ and $\sim 0.1-0.3 \mathrm{Jy}$. The respective flux ratios are consistent with the ones in the opti$\mathrm{cal} / \mathrm{UV}$ and X-rays. The mm radio flux density of $3 \mathrm{C} 345$ was at least a factor of 7 higher than for NRAO 512 and B3 1640+396.

No significant excess of $\gamma$-ray emission from NRAO 512 was observed during the first 15-months of the Fermi-LAT operation. At the end of 2009, NRAO 512 showed an increasing trend in its $15 \mathrm{GHz}$ VLBI radio flux observed by the MOJAVE team (Lister et al. 2009) ${ }^{7}$. Indeed, the radio flux reached a low activity level around May 2009 and more than doubled until about July 2010 with the mm-radio flux density reaching a peak value of over 0.6 Jy in August 2010. A similar trend was observed in optical emission. Within one year, the optical flux increased by a factor of $\sim 2.2$ (see Table 3 ). In X-rays a flux increase of at least 20-30\% was observed, but because of the low statistics (less than 200 photons) it is difficult to compare the observations. An increased number of $\gamma$-ray photons

\footnotetext{
7 http://www.physics.purdue.edu/MOJAVE/sourcepages/ 1638+398. shtml
}

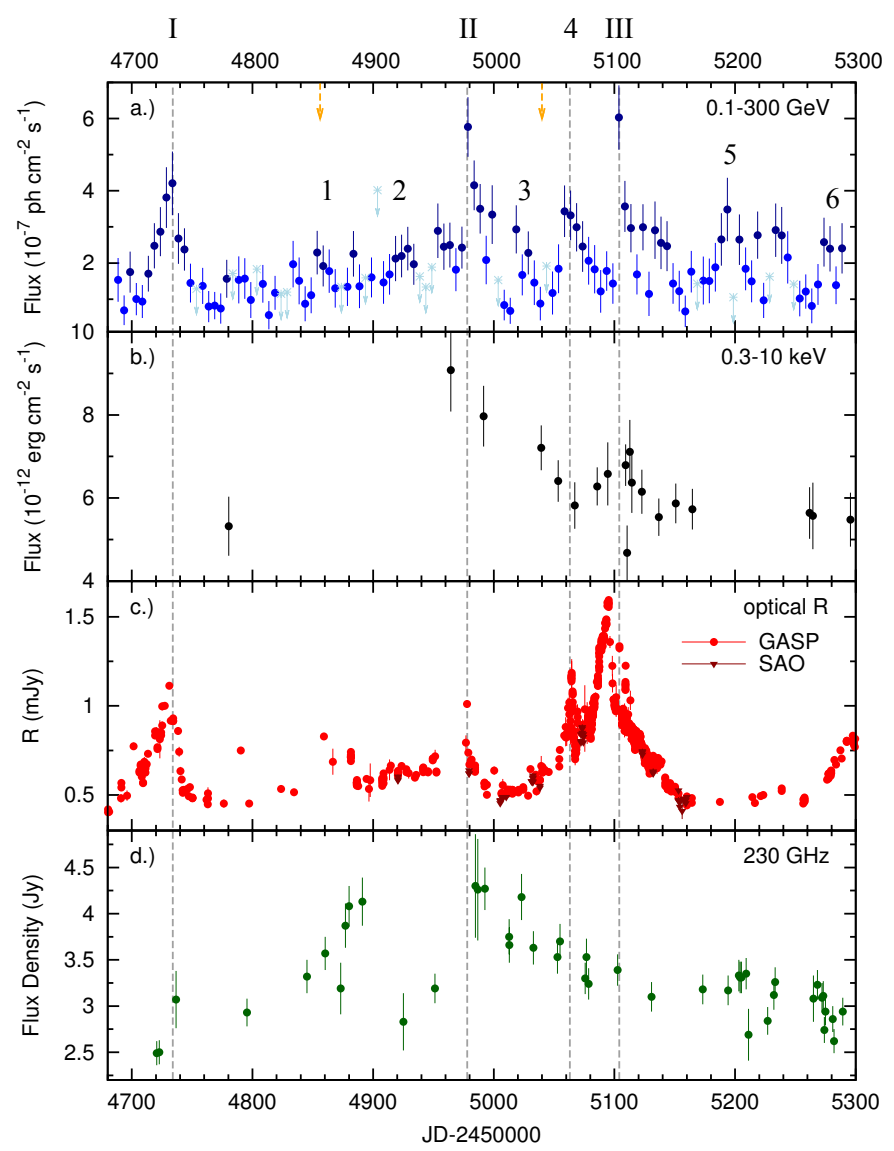

Fig. 4. Multiwavelength light curves of 3C 345 for the 20-month period discussed in this paper, from top to bottom for: a) $\gamma$ rays observed by Fermi-LAT between 0.1 and $300 \mathrm{GeV}$, values (filled circles) are five-day integrated, $2 \sigma$ upper limits for five-day integrations are shown as lightblue crosses with downward arrows where $T S<5$. Dark-blue filled circles have a $T S>25$, the others are in the range $5<T S \leq 25$. In contrast to Fig. 3, the $\gamma$-ray emission was fitted by a single power-law component placed at the position of 3C 345. b) X-rays observed by Swift XRT between 0.3 and $10 \mathrm{keV}$. c) Optical ( $R$-band) observations performed by GASP and SAO. d) Millimeter radio observations by the SMA at $230 \mathrm{GHz}$. The two orange downward arrows on top indicate the observation epoch for which a new feature was detected in the pc-scale radio jet of 3C 345, see Sect. 3.4 and Schinzel et al. (2010). Three dominant flare events of high significance observed on two-day time scales were labeled with I-III. Several weaker events were identified and labeled with numbers from 1-6.

was detected spatially consistent with NRAO 512 during the last quarter of the 20-month period. The excess is not high enough to affect the short-term bins of the light curve of 3C 345 significantly, however, as was shown in Sect. 3.1, the integrated 20-month dataset made it possible to detect a second point source west of 1FGL J1642.5+3947, which is consistent with the radio position of NRAO 512 (see Fig. 1). Altogether this identifies NRAO 512 as a $\gamma$-ray source.

\subsection{Multi-wavelength analysis of $\gamma$-ray emission from $3 C 345$}

During 2009 nine optical observatories, listed in Sect. 2.3, regularly monitored 3C 345, mainly as part of the GASP-WEBT program. This provided a densely sampled optical $R$-band light curve (Fig. 4). Visual comparison of the $\gamma$-ray and optical light curves indicates a likely correspondence between at least 
A\&A 532, A150 (2011)

Table 5. Characteristics of prominent $\gamma$-ray events during the first 20 months of the Fermi-LAT observations.

\begin{tabular}{lccccccc}
\hline \hline $\begin{array}{l}t_{\text {peak }} \\
\mathrm{JD}(\dagger)\end{array}$ & $\begin{array}{c}t_{\text {start }} \\
\mathrm{JD}(\dagger)\end{array}$ & $\begin{array}{c}t_{\text {stop }} \\
\mathrm{JD}(\dagger)\end{array}$ & $\begin{array}{c}\Delta t \\
\text { days }\end{array}$ & $\begin{array}{c}\Delta t_{\text {rise }} \\
\text { days }\end{array}$ & $\begin{array}{c}\Delta t_{\text {fall }} \\
\text { days }\end{array}$ & $\begin{array}{c}S_{\gamma}^{\max } \\
10^{-7} \frac{\mathrm{ph}}{\mathrm{cm}^{2} \mathrm{~s}}\end{array}$ & $(*)$ \\
\hline 4734 & 4709 & 4754 & 45 & 25 & 20 & $5.5 \pm 1.5$ & $\mathrm{I}$ \\
4856 & 4844 & 4874 & 30 & 12 & 18 & $3.6 \pm 1.1$ & 1 \\
4916 & 4904 & 4944 & 40 & 12 & 28 & $4.0 \pm 1.2$ & 2 \\
$4978 / 4998$ & 4969 & 5014 & 45 & 9 & 16 & $5.7 \pm 1.3 / 5.4 \pm 1.5$ & $\mathrm{II}$ \\
5020 & 5014 & 5044 & 30 & 6 & 24 & $4.5 \pm 1.2$ & 3 \\
$5056 / 5070$ & 5044 & 5089 & 45 & 12 & 19 & $4.4 \pm 1.4 / 4.5 \pm 1.2$ & 4 \\
5104 & 5099 & 5119 & 20 & 5 & 15 & $9.1 \pm 1.6$ & $\mathrm{III}$ \\
5194 & 5169 & 5214 & 45 & 25 & 20 & $4.7 \pm 1.4$ & 5 \\
5278 & 5264 & & & 14 & & $3.6 \pm 1.1$ & 6 \\
\hline Average values: & & & 38 & 13 & 20 & $5.0 \pm 0.5$ & \\
\hline
\end{tabular}

Notes. $t_{\text {peak }}$ - in units of JD( $\left.\dagger\right)=$ JD-2 450000 , peak value was obtained from the two-day integrated light curve, all values have an error of \pm 1 day; $t_{\text {start }}, t_{\text {stop }}-$ in units of $\mathrm{JD}(\dagger)=\mathrm{JD}-2450000$, all values have an error of \pm 3 days; $\Delta t, \Delta t_{\text {rise }}, \Delta t_{\text {fall }}-$ in units of days, all values with error of \pm 4 days; $S_{\gamma}^{\max }$ - two-day integrated flux value of the $\gamma$-ray event peak value; $(*)$ labels are split into two sub-categories according to their peak flux and significance of detection, denoted by Roman and Arab numbers, see Fig. 4 for details.

four $\gamma$-ray/optical flare events: Flare I, II, 4 and III. With the addition of X-ray and mm-radio monitoring data, the following aspects are of particular interest:

1. A possible fast optical brightening of at least $0.3 \mathrm{mag}$ with a duration of only a few days (Fig. 4) was observed (JD 2454978), coincident with the peak of Flare II. Unfortunately, the optical monitoring had a gap between JD 2454953 and JD2454977. At the same time the mm radio light curve showed a maximum of around $4.3 \mathrm{Jy}$. Moreover, a high X-ray flux was observed in the same period of the Flare II, followed by a decrease similar to what was observed in $\gamma$ and radio bands. Even if the maximum X-ray flux was observed two weeks before Flare II, the $\gamma$-ray event lacks strictly simultaneous X-ray observations, thus we cannot exclude that a simultaneous X-ray flare was missed.

2. The broad optical flaring episode observed around September/October 2009 had a total duration of about five months, with shorter timescale sub-structures that correspond to activity variations observed also at $\gamma$ and X-rays. The peak in the optical was observed on September 19th, 2009 (JD 2455 094), and had no similarity in profile with events at other wavelengths (see Fig. 4).

3. Ten days after the peak of the optical light curve, the strong $\gamma$-ray Flare III was observed. The optical peak was followed by fast optical variability at a significant amplitude of 0.3-0.5 mag, with an elevated magnitude observed on September 29th on the same day as $\gamma$-ray Flare III.

4. Flares 1 and 3 can be related to the appearance of new features in the pc-scale radio jet of $3 \mathrm{C} 345$ at $43 \mathrm{GHz}$ (see Schinzel et al. 2010).

5. All but Flares 5 and 6 occur before the significant excess of $\gamma$-ray emission was observed near NRAO 512. However, for at least Flare 6, a correspondence in optical variability was found for 3C 345; unfortunately, a lack of multiwavelength data around Flare 5 makes it impossible to identify it with either 3C 345 or NRAO 512.

To quantify the cross-correlation between light curves, a discrete correlation was calculated (DCF; Edelson \& Krolik 1988) using the 20-month Fermi-LAT light curve and the optical $R$-band light curve between JD 2454680 (August 1st, 2008) and 2455299 (April 12th, 2010), as well as the radio SMA light curve between JD 2454721 (September 11th, 2008) and 2455289 (April 2nd, 2010). Figure 5 (top) plots the resulting
DCF comparing optical and $\gamma$-ray light curves using a five-day $\gamma$-ray integration and a ten-day timelag binning. This yields a number of distinct correlation peaks, of which the two most significant are discussed. In the first peak the optical leads the $\gamma$-ray flux by $(15 \pm 10)$ days with a correlation coefficient of $0.44 \pm 0.05$. The second peak corresponds to the optical flux lagging the $\gamma$-rays by $110 \pm 5$ days with a correlation coefficient of $0.77 \pm 0.06$. To test the robustness of these peaks in the DCF, we artificially removed the points corresponding to the peak $\gamma$-ray flux from Flares II and III and recalculated the DCF. The same behavior was observed, with the $\gamma$-ray flux leading the optical by 20 days and lagging by 110 days. This shows that the results obtained do not solely depend on these two events. Finally, the DCF between the $\gamma$-ray and the SMA light curve of 3C 345 was calculated, which yielded a noisy distribution of correlation peaks (also see Fig. 5). The strongest peak indicates that the radio emission leads the $\gamma$ rays by $120 \pm 5$ days with a correlation coefficient of $0.83 \pm 0.30$.

The statistical significance of the cross-correlation peaks was investigated using Monte Carlo simulations, following Chatterjee et al. (2008) and Max-Moerbeck et al. (2010) and assuming that the noise properties of the light curves can be described by a power-law power spectral density (PSD; $\propto 1 / f^{-\beta}$ ) with an exponent that depends on the particular energy band of the emission.

In order to estimate the significance of the $\gamma$-ray/optical cross correlation, an attempt was made to calculate and characterize the PSD shape from the presented data directly, despite the limited length (20 months) and sampling ( $>1$ day) of the light curves. For the optical and $\gamma$-ray data, the light curves available support a range of time scales corresponding to about one decade in the frequency domain. The respective range is even smaller for the more sparsely sampled radio data. The PSDs were calculated following Sect. 3.1 in Uttley et al. (2002). The resulting powerlaw slopes, $\beta$, are in the range of 1.2-0.7. Values of $\beta \gg 2.0$ are not consistent with the calculated PSD shapes and can be ruled out. However, Uttley et al. (2002) demonstrated that aliasing effects add to the power spectral slopes and could potentially flatten the observed power spectrum. The relatively high noise and short time span of the data makes this range of $\beta$ rather unreliable. Monte Carlo simulations for these values of $\beta$ yield extremely high cross-correlation significances of $>99.999 \%$ for the $\gamma$-ray/optical light curves and $>99.98 \%$ for the $\gamma$-ray/radio case. It should be noted that a reliable determination of the PSD from 


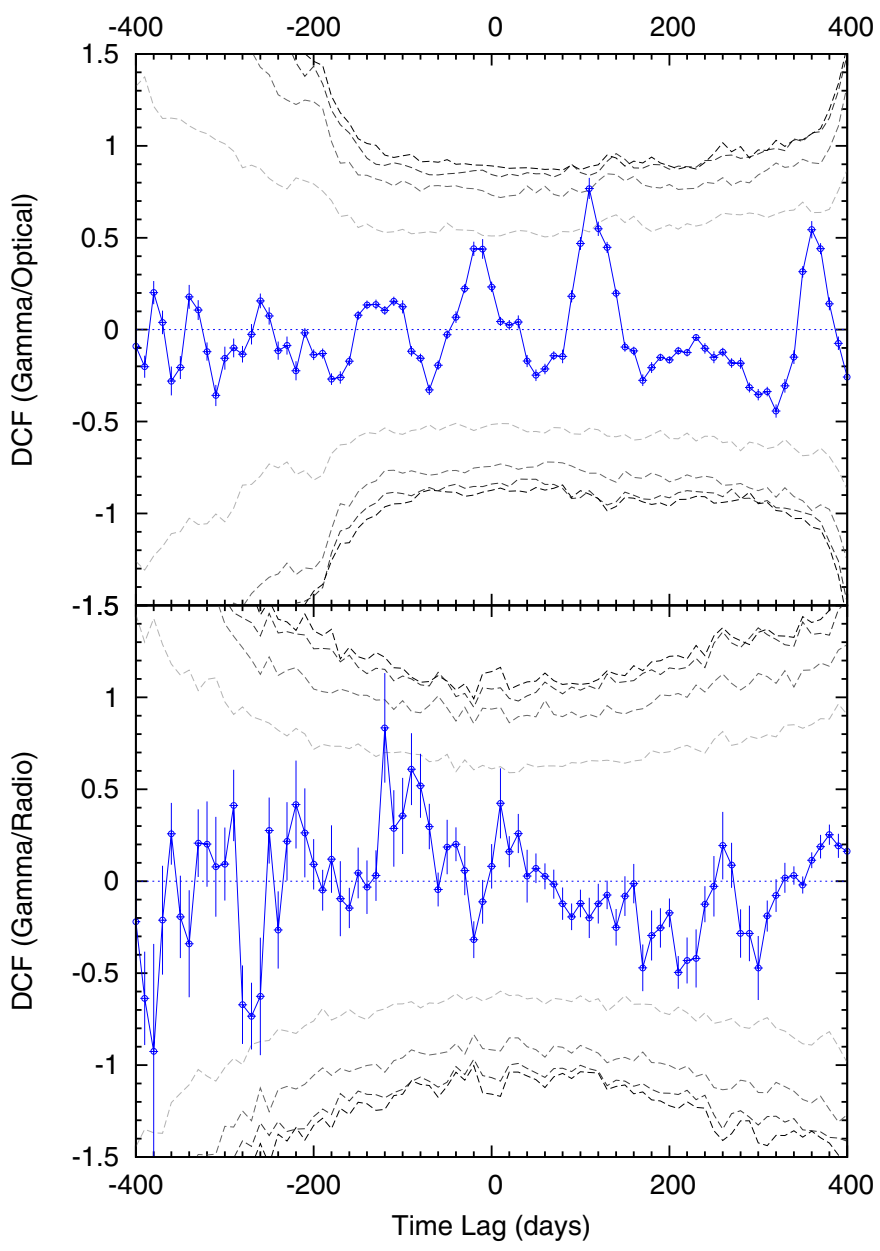

Fig. 5. Correlation between the five-day integrated $\gamma$-ray light curve and the optical (top) and radio (bottom) light curves of 3C 345. The correlation is obtained by calculating the discrete correlation function (DCF) with ten-day binning. A positive time-lag corresponds to the $\gamma$-ray variations preceding the variations in the other two bands. The significance of the correlation is illustrated by $3 \sigma(99.7 \%)$ significance contours calculated from simulated, uncorrelated data with different slopes, $\beta$, of the power spectral density (PSD). The contours are drawn for the PSD slopes $\beta=1.0,1.5,2.0$, and 2.5 (indicated by progression of colors, from light gray to black). For the simulated data, values of the correlation coefficient exceeding \pm 1 are an inherent feature of the method, resulting from differences in overlap of the time series at different time lags (cf. Edelson \& Krolik 1988; Timmer \& Koenig 1995, and the description of the applied method in Sect. 3.4).

observational data is usually done with a much larger dynamic range (e.g. covering 4-5 orders of magnitude in the frequency scale), which is also the case in Uttley et al. (2002). The radio, optical and $\gamma$-ray data discussed in our paper do not provide such an extended coverage in the frequency space. We adopt therefore a more cautious approach, exploring a range of power-law indices based on published values.

In the $\gamma$-ray regime $\beta_{\gamma}=1.7 \pm 0.3$ for BL Lacs and $\beta_{\gamma}=$ $1.4 \pm 0.1$ for flat spectrum radio quasars (Abdo et al. 2010c). Instead, for $3 \mathrm{C} 279$, it has been found that $\beta_{\text {radio }}=2.3 \pm 0.5$ at $14.5 \mathrm{GHz}$ and $\beta_{\text {optical }}=1.7 \pm 0.3$ in the $R$-band (Chatterjee et al. 2008). More recently Chatterjee et al. (2011) found an average optical power-law index of $\beta_{\text {optical }}=1.6 \pm 0.3$ for a sample of six blazars in the $R$-band. Simulated light curves with PSD power-law indices in these ranges can provide reliable estimates on the significance of the cross-correlations.
The simulations use light curves of the same length as the data and simulated up to a maximum frequency of 1 day $^{-1}$ using the method described by Timmer \& Koenig (1995). Each case uses 10000 pairs of light curves, which are sampled and crosscorrelated in the same fashion as the observed light curves. From these simulated data the distribution of correlation coefficients for each time bin can be obtained for the case of non correlated time series. Figure 5 presents the $3 \sigma(99.7 \%)$ significance levels for all tested PSD shape combinations.

The $\gamma$-ray/optical correlation has a significance of higher than $3 \sigma(99.7 \%)$ for the peak at a timelag of 110 days in most of the tested cases. It is the most significant peak in the DCF and it has a moderately high confidence level (>98.5\%) even for the least significant case $\left(\beta_{\gamma}=2.5\right.$ and $\beta_{\text {optical }}=2.5$; highest dashed line in the top panel of Fig. 5) among the $\beta$ values considered. The $\gamma$-ray/optical peak at a timelag of about -20 days has a much lower confidence level $(>67.6 \%)$ in the least significant case and it remains below the $3 \sigma$ level at all times. The $\gamma$-ray/radio cross-correlation at timelag -120 days has a confidence level of $>90.7 \%$ in the least significant case $\left(\beta_{\gamma}=2.5\right.$ and $\left.\beta_{\text {radio }}=3.0\right)$.

The source 3C 345 is by far the brightest of the three quasars in the $\gamma$-ray region studied during the presented time period. In all bands it was consistently brighter than NRAO 512 and B3 1640+396. The improved Fermi position combined with multi-wavelength activity and a moderate significance of correlated optical $/ \gamma$-ray variability, using a plausible range of $\beta$ in the $\gamma$-ray and optical PSDs, strongly suggest an identification of $3 \mathrm{C} 345$ as $\gamma$-ray source and the main contributor to the observed $\gamma$-ray flux in 1FGL J1642.5+3947.

\section{Discussion and summary}

Properties of the $\gamma$-ray emitting region reported in the first threemonth bright source list (Abdo et al. 2009a) as well as in the first 11-month Fermi-LAT source catalog (Abdo et al. 2010a,b) were compared to the 20-month dataset presented here. Localizations of the sources in this region were improved over the previously reported values, leading to the detection of two $\gamma$-ray point sources, one consistent with 1FGL J1642.5+3947 and a new $\gamma$-ray source $\sim 0.4^{\circ}$ west of it. 3C 345 and NRAO 512 were identified as the counterparts for these $\gamma$-ray sources. Their $\gamma$-ray spectra have a spectral slope of $\Gamma=2.4-2.5$ typical for that of flat spectrum radio quasars (Abdo et al. 2010b). No significant spectral break was observed in the spectrum of either source, unlike in the case of 3C 454.3 (Ackermann et al. 2010), however, the statistics at the highest energies are poor, and the presence of a spectral break at $E>10 \mathrm{GeV}$ is not ruled out.

The $\gamma$-ray emission from 3C 345 was identified based on an improved $\gamma$-ray counterpart localization and multiwavelength activity including correlations of moderate significance found between the optical and radio variability, using a plausible range of $\beta$ in the $\gamma$-ray and optical PSDs, and major $\gamma$-ray events observed by Fermi-LAT during its first 20 months of operation. EGRET observed 3C 345 between 1991 and 1994 during a time of enhanced radio activity. An upper limit of $2.5 \times 10^{-7} \mathrm{ph} \mathrm{cm}^{-2} \mathrm{~s}^{-1}$ for the viewing period September 12th-19th, 1991, was reported (Fichtel et al. 1994). However, Casandjian \& Grenier (2008) report the detection of a $\gamma$-ray source that could be consistent with 3C 345 in the period April 23rd-May 7th, 1996 with a $\gamma$-ray flux of $(3.5 \pm 0.8) \times 10^{-7} \mathrm{ph} \mathrm{cm}^{-2} \mathrm{~s}^{-1}$, but it was noted that the association with 3 C 345 remains unclear because of a possible 
confusion with Mrk501. In the Fermi-era, with an increased sensitivity and the possibility of long-term integrations, 3C 345 was already detected during the first three months of operation at a level of $\sim 18 \sigma$ and after 20 months it reached over $30 \sigma$. However, owing to the unclear association of 3C 345 with EGR J1642+3940, the three-month data were falsely associated with CLASS J1641+3035 (B3 1640+396), which, as shown in this paper, did not produce a significant excess of $\gamma$-ray emission above the detection threshold of Fermi-LAT. Thus the simplest explanation for the previous non-detection of 3C 345 is that its emission was just below or at the detection limit of EGRET.

These findings end the decade-long debate whether 3C 345 is $\gamma$-ray loud (Unwin et al. 1997). The identification of 1FGL J1642.5+3947 with 3C 345 is further supported by the connection of radio-emission of the parsec-scale jet with the observed $\gamma$-ray emission (Schinzel et al. 2010). Here not only individual flares were correlated with radio events, but an unambigious long-term trend in $\gamma$-ray emission was observed, which matched the trend of the radio flux density light curve of the inner jet at $43 \mathrm{GHz}$. The trend and correlated radio events relate to a distance of $\sim 10 \mathrm{pc}$ in the source frame and questions existing scenarios that place the $\gamma$-ray emission at sub-pc distances from the central engine (e.g., Tavecchio et al. 2010). This supports the arguments given by Unwin et al. (1997) that the observed $\gamma$-ray emission is related to the relativistic jet on pc scales.

The cross-correlation found between $\gamma$-ray and optical light curves at 110 days had a moderate confidence level of $>98.5 \%$, whereas the lag at -20 days had a confidence level of only $>67.6 \%$ using a plausible range of $\beta$ in the $\gamma$-ray and optical PSDs. If typical values for the power spectral density (PSD) shapes are assumed $\left(\beta_{\gamma}=1.5\right.$ and $\beta_{\text {optical }}=1.5$; second to lowest dashed line in the top panel of Fig. 5), the confidence level remains low at $>89.3 \%$. The weak correlation found between the $\gamma$-ray and $230 \mathrm{GHz}$ radio light curves had a worst-case confidence level of $>89.3 \%$. Assuming a possibly more typical combination of PSD shapes $\left(\beta_{\gamma}=1.5\right.$ and $\left.\beta_{\text {radio }}=2.0\right)$, a confidence level of $>96.1 \%$ is obtained. If this weak correlation holds, it could suggest a connection between the radio emission and production of $\gamma$-rays. A timelag of $\sim-120$ days suggests the mm-radio emission precedes the $\gamma$-rays. Pushkarev et al. (2010) showed for a larger sample of radio-loud quasars that on average the $15 \mathrm{GHz}$ radio emission lags $\sim 1.2$ months behind $\gamma$-rays. The result presented here indicates that between $15 \mathrm{GHz}$ and $230 \mathrm{GHz}$ it is possible to directly observe the $\gamma$-ray emitting region, as already implied by Unwin et al. (1997), which supports the argument of $\gamma$-ray production in the relativistic jet on pc scales. However, in contrast to these, a more confident cross-correlation result of $\gamma$-rays leading by $\sim 80$ and $\sim 50$ days was recently obtained for two prominent BL Lac type objects, OJ 287 (Agudo et al. 2011a) and AO 0235+164 (Agudo et al. $2011 b)$, respectively. Doubts on the indicated radio/ $\gamma$-ray correlation of 3 C 345 remain in this problem and should be investigated more thoroughly with a longer and denser sampled lightcurve at $\mathrm{mm}$ wavelengths.

The $\gamma$-ray emission from NRAO 512, a high-redshift quasar, was identified based on a correlated increase in multiwavelength emission from radio up to $\mathrm{GeV}$ energies between 2009 and 2010. At monthly timescales the source started to be significant around November 2009. This increase of $\gamma$-ray flux led to the first detection of the now identified new $\gamma$-ray counterpart west of 3C 345 . The LAT flux quoted in this paper for the first 15 months was below the EGRET detection threshold, offering a plausible explanation of why EGRET did not see emission from NRAO 512, except possibly during a $\gamma$-ray flare event.
The radio, optical and X-ray flux ratios between 3C 345, NRAO 512 and B3 1640+396 could be used to gauge their individual $\gamma$-ray photon contributions and might be able to provide an indication of false associations for $\gamma$-ray photons from this part of the sky. Multiwavelength monitoring, in addition to the continuous all-sky monitoring by Fermi-LAT, will help to improve the localization of $\gamma$-ray emission from this region and is necessary for a deeper variability study for any of these sources in the future. Ultimately this might lead to the detection of $\gamma$-ray emission from B3 1640+396 as well.

Acknowledgements. The authors would like to thank Ann Wehrle, Benoit Lott, Seth Digel, Gino Tosti, Dave Thompson, and Greg Taylor for their helpful comments and useful discussions, which improved the quality of this manuscript. We thank the A\&A referee for extensive comments. The FermiLAT Collaboration acknowledges generous ongoing support from a number of agencies and institutes that have supported both the development and the operation of the LAT as well as scientific data analysis. These include the National Aeronautics and Space Administration and the Department of Energy in the United States, the Commissariat à l'Energie Atomique and the Centre National de la Recherche Scientifique/Institut National de Physique Nucléaire et de Physique des Particules in France, the Agenzia Spaziale Italiana and the Istituto Nazionale di Fisica Nucleare in Italy, the Ministry of Education, Culture, Sports, Science and Technology (MEXT), High Energy Accelerator Research Organization (KEK) and Japan Aerospace Exploration Agency (JAXA) in Japan, and the K. A. Wallenberg Foundation, the Swedish Research Council and the Swedish National Space Board in Sweden. Additional support for science analysis during the operations phase is gratefully acknowledged from the Istituto Nazionale di Astrofisica in Italy and the Centre National d'Etudes Spatiales in France. Frank Schinzel and Kirill Sokolovsky were supported for this research through a stipend from the International Max Planck Research School (IMPRS) for Astronomy and Astrophysics at the Universities of Bonn and Cologne. M. Villata organized the optical-to-radio observations by GASPWEBT as the president of the collaboration. Additional support for science analysis during the operations phase is gratefully acknowledged from the Istituto Nazionale di Astrofisica in Italy and the Centre National d'Études Spatiales in France. The St. Petersburg University team acknowledges support from Russian RFBR foundation via grant 09-02-00092. Acquisition of the MAPCAT data is supported in part by the Spanish Ministry of Science and Innovation and the Regional Government of Andalucía through grants AYA2007-67626C03-03 and P09-FQM-4784, respectively. The Abastumani team acknowledges financial support by the Georgian National Science Foundation through grant GNSF/ST08/4-404. A. Marscher and S. Jorstad received support for this work from NASA grants NNX08AV65G, NNX08AV61G, and NNX09AT99G, and National Science Foundation grant AST-0907893 to Boston University. The Submillimeter Array is a joint project between the Smithsonian Astrophysical Observatory and the Academia Sinica Institute of Astronomy and Astrophysics and is funded by the Smithsonian Institution and the Academia Sinica. This paper is partly based on observations carried out at the German-Spanish Calar Alto Observatory, which is jointly operated by the MPIA and the IAACSIC. This research has made use of NASA's Astrophysics Data System, the SIMBAD database, operated at CDS, Strasbourg, France and the NASA/IPAC Extragalactic Database (NED) which is operated by the JPL, Caltech, under contract with NASA. This research has made use of data from the MOJAVE database that is maintained by the MOJAVE team (Lister et al. 2009).

\section{References}

Abazajian, K. N., Adelman-McCarthy, J. K., Agüeros, M. A., et al. 2009, ApJS, 182,543

Abdo, A. A., Ackermann, M., Ajello, M., et al. 2009a, ApJS, 183, 46 Abdo, A. A., Ackermann, M., Ajello, M., et al. 2009b, ApJ, 700, 597 Abdo, A. A., Ackermann, M., Ajello, M., et al. 2009c, Phys. Rev. D, 80, 122004 Abdo, A. A., Ackermann, M., Ajello, M., et al. 2010a, ApJS, 188, 405 Abdo, A. A., Ackermann, M., Ajello, M., et al. 2010b, ApJ, 715, 429 Abdo, A. A., Ackermann, M., Ajello, M., et al. 2010c, ApJ, 722, 520 Ackermann, M., Ajello, M., Baldini, L., et al. 2010, ApJ, 721, 1383 Agudo, I., Jorstad, S. G., Marscher, A. P., et al. 2011a, ApJ, 726, L13 Agudo, I., Marscher, A. P., Jorstad, S. G., et al. 2011b, ApJ, 735, L10 Atwood, W. B., Abdo, A. A., Ackermann, M., et al. 2009, ApJ, 697, 1071 Bessell, M. S., Castelli, F., \& Plez, B. 1998, A\&A, 333, 231 Brinkmann, W., Siebert, J., Reich, W., et al. 1995, A\&AS, 109, 147 Burrows, D. N., Hill, J. E., Nousek, J. A., et al. 2005, Space Sci. Rev., 120, 165 Bychkova, V. S., Kardashev, N. S., Vlasyuk, V. V., \& Spiridonova, O. I. 2004, Astron. Rep., 48, 840

Cardelli, J. A., Clayton, G. C., \& Mathis, J. S. 1989, ApJ, 345, 245 
Casandjian, J.-M., \& Grenier, I. A. 2008, A\&A, 489, 849

Cash, W. 1979, ApJ, 228, 939

Chatterjee, R., Jorstad, S. G., Marscher, A. P., et al. 2008, ApJ, 689, 79

Chatterjee, R., Bailyn, C., Bonning, E. W., et al. 2011 [arXiv: 1101. 3815]

Edelson, R. A., \& Krolik, J. H. 1988, ApJ, 333, 646

Fey, A. L., Ma, C., Arias, E. F., et al. 2004, AJ, 127, 3587

Fichtel, C. E., Bertsch, D. L., Chiang, J., et al. 1994, ApJS, 94, 551

Gehrels, N., Chincarini, G., Giommi, P., et al. 2004, ApJ, 611, 1005

González-Pérez, J. N., Kidger, M. R., \& Martín-Luis, F. 2001, AJ, 122, 2055

Hartman, R. C., Bertsch, D. L., Bloom, S. D., et al. 1999, ApJS, 123, 79

Kalberla, P. M. W., Burton, W. B., Hartmann, D., et al. 2005, A\&A, 440, 775

Kataoka, J., Mattox, J. R., Quinn, J., et al. 1999, ApJ, 514, 138

Kollgaard, R. I., Wardle, J. F. C., \& Roberts, D. H. 1989, AJ, 97, 1550

Kovalev, Y. Y., Nizhelsky, N. A., Kovalev, Y. A., et al. 1999, VizieR Online Data Catalog, 413, 90545

Larionov, V. M., Villata, M., Raiteri, C. M., et al. 2009, The Astronomer's Telegram, 2222, 1

Lister, M. L., Aller, H. D., Aller, M. F., et al. 2009, AJ, 137, 3718

Lobanov, A. P., \& Zensus, J. A. 1999, ApJ, 521, 509

Marziani, P., Sulentic, J. W., Dultzin-Hacyan, D., Calvani, M., \& Moles, M. 1996, ApJS, 104, 37

Mattox, J. R., Bertsch, D. L., Chiang, J., et al. 1996, ApJ, 461, 396

Max-Moerbeck, W., Richards, J. L., Pavlidou, V., et al. 2010, in Fermi meets Jansky - AGN in Radio and Gamma-Rays, ed. T. Savolainen, E. Ros, W. P. Porcas, \& J. A. Zensus, 77

Myers, S. T., Jackson, N. J., Browne, I. W. A., et al. 2003, MNRAS, 341, 1

Poole, T. S., Breeveld, A. A., Page, M. J., et al. 2008, MNRAS, 383, 627
Pushkarev, A. B., Kovalev, Y. Y., Lister, M. L., \& Savolainen, T. 2009, A\&A, 507, L33

Pushkarev, A. B., Kovalev, Y. Y., \& Lister, M. L. 2010, ApJ, 722, L7

Rando, R., et al. 2009 [arXiv: 0907.0626]

Reyes, L. C., \& Cheung, C. C. 2009, The Astronomer's Telegram, 2226, 1

Rolke, W. A., López, A. M., \& Conrad, J. 2005, NIM A, 551, 493

Roming, P. W. A., Kennedy, T. E., Mason, K. O., et al. 2005, Space Sci. Rev., 120,95

Roming, P. W. A., Koch, T. S., Oates, S. R., et al. 2009, ApJ, 690, 163

Schinzel, F. K., Lobanov, A. P., Jorstad, S. G., et al. 2010, in Fermi meets Jansky - AGN in Radio and Gamma-Rays, ed. T. Savolainen, E. Ros, W. P. Porcas, \& J. A. Zensus, 175

Schlegel, D. J., Finkbeiner, D. P., \& Davis, M. 1998, ApJ, 500, 525

Smith, P. S., Balonek, T. J., Heckert, P. A., Elston, R., \& Schmidt, G. D. 1985, AJ, 90,1184

Smith, J. A., Tucker, D. L., Kent, S., et al. 2002, AJ, 123, 2121

Stickel, M., Fried, J. W., \& Kuehr, H. 1989, A\&AS, 80, 103

Tavecchio, F., Ghisellini, G., Bonnoli, G., \& Ghirlanda, G. 2010, MNRAS, 405, L94

Thompson, D. J., Bertsch, D. L., Fichtel, C. E., et al. 1993, ApJS, 86, 629

Timmer, J., \& Koenig, M. 1995, A\&A, 300, 707

Unwin, S. C., Wehrle, A. E., Urry, C. M., et al. 1994, ApJ, 432, 103

Unwin, S. C., Wehrle, A. E., Lobanov, A. P., et al. 1997, ApJ, 480, 596 Uttley, P., McHardy, I. M., \& Papadakis, I. E. 2002, MNRAS, 332, 231

Villata, M., Raiteri, C. M., Larionov, V. M., et al. 2008, A\&A, 481, L79

Villata, M., Raiteri, C. M., Gurwell, M. A., et al. 2009, A\&A, 504, L9

Wilks, S. S. 1938, Ann. Math. Stat., 9, 60 